\title{
Identification of Potentially Therapeutic Target Genes of Hepatocellular Carcinoma
}

\author{
Chengzhang $\mathrm{Li}^{1}$ and Jiucheng $\mathrm{Xu}{ }^{1,2, *}$ \\ 1 College of Life Science, Henan Normal/ University, Xinxiang 453007, China; lee125@126.com \\ 2 Engineering Lab of Intelligence Business \& Internet of Things, College of Computer and Information \\ Engineering, Henan Normal University, Xinxiang 453007, China \\ * Correspondence: xjc@htu.cn
}

Received: 22 January 2020; Accepted: 5 February 2020; Published: 7 February 2020

check for updates

\begin{abstract}
Background: Hepatocellular carcinoma (HCC) is a major threat to public health. However, few effective therapeutic strategies exist. We aimed to identify potentially therapeutic target genes of HCC by analyzing three gene expression profiles. Methods: The gene expression profiles were analyzed with GEO2R, an interactive web tool for gene differential expression analysis, to identify common differentially expressed genes (DEGs). Functional enrichment analyses were then conducted followed by a protein-protein interaction (PPI) network construction with the common DEGs. The PPI network was employed to identify hub genes, and the expression level of the hub genes was validated via data mining the Oncomine database. Survival analysis was carried out to assess the prognosis of hub genes in HCC patients. Results: A total of 51 common up-regulated DEGs and 201 down-regulated DEGs were obtained after gene differential expression analysis of the profiles. Functional enrichment analyses indicated that these common DEGs are linked to a series of cancer events. We finally identified 10 hub genes, six of which (OIP5, ASPM, NUSAP1, UBE2C, CCNA2, and KIF20A) are reported as novel HCC hub genes. Data mining the Oncomine database validated that the hub genes have a significant high level of expression in HCC samples compared normal samples ( $t$-test, $p<0.05)$. Survival analysis indicated that overexpression of the hub genes is associated with a significant reduction $(p<0.05)$ in survival time in HCC patients. Conclusions: We identified six novel HCC hub genes that might be therapeutic targets for the development of drugs for some HCC patients.
\end{abstract}

Keywords: hepatocellular carcinoma; hub gene; PPI network; MCC algorithm; gene expression profile

\section{Introduction}

Hepatocellular carcinoma (HCC), a major threat to public health, is the fifth most common cancer worldwide, causes about one million deaths each year [1,2]. HCC mortality rates have risen over the last few decades in most countries [3]. HCC can be managed by the following main treatments: liver transplantation or resection [4], transcatheter arterial chemoembolization (TACE) [5], radiofrequency ablation (RFA) [6], transarterial radioembolization (TARE) [7], and targeted systemic chemotherapy [8]. Surgical treatment is normally considered standard potentially curative treatment for early-stage HCC [9]. However, this is not a comprehensive HCC strategy, as most of the patients diagnosed at advanced stage have a poor prognosis [2]. Despite the numerous efforts to treat of HCC, the five-year recurrence rate remains high $(\sim 60 \%)$ after surgical treatment [10]. Therefore, the need is urgent to develop more effective treatments that can improve the long-term survival rate of HCC patients.

The pathogenesis of liver cancer is complicated [11]. Multiple risk factors, such as age and race [12], hepatitis $C$ virus [13], and hepatitis B virus [14], are considered to contribute to the abnormal expression of genes associated with initiation or progression of HCC. One of the strategies under 
investigation for the treatment of $\mathrm{HCC}$ is to discover essential genes contributing to the initiation or progression of HCC. Recent evidence shows that a variety of genes are crucial regulators of HCC. For instance, elevation of chemokine (C-X-C motif) ligand 17 (CXCL17) expression was demonstrated to enhance HCC cell metastasis and suppress autophagy via the LKB1 (a serine/threonine kinase)-AMPK (a central metabolic sensor) pathway [15]. The analysis of tumor necrosis factor- $\alpha$ (TNF- $\alpha$ ) expression in 62 HCC patients indicated that TNF- $\alpha$ may serve as a potential HCC therapeutic target to overcome sorafenib resistance [16]. Overexpression of hepatic cyclooxygenase-2 in mice was reported to induce spontaneous formation of HCC [17]. Overall, many genes have abnormal expressions in HCC. The HCC-associated genes, along with a subset of their neighbors, are generally developed into a gene interaction network. Some researchers are interested in studying HCC from the perspective of gene interaction networks rather than assess the genes individually. The availability of multiple network topological analytic methods has enabled the identification of a number of hub genes with important pathological significance [18-20].

HCC forms a highly heterogeneous tumor, and various alterations in gene expression can promote the initiation and progression of this cancer. Zhang et al. found the top 10 hub genes for HCC are ALB, TGFB1, GMPS, ACACA, KRAS, EGFR, STAT3, ERBB2, BCL2, and CD8A [11]. In contrast, another study showed that JUN, EGR1, MYC, and CDKN1A are hub genes of HCC [21]. Heterogenicity may contribute to the differences in these two studies. Most of the previous studies were performed with only one micro-array gene expression profile which may not fully detect all potentially HCC hub genes. Attempting to find more representative genes of HCC, we integrally analyzed three micro-array gene expression profiles to detect genes that may serve as therapeutic target genes of HCC.

\section{Materials and Methods}

\subsection{Data Selection}

Three micro-array gene expression profiles (GSE87630, GSE84598, and GSE89377) were selected from the Gene Expression Omnibus (GEO) repository [11]. GSE87630 is based on the GPL6947 platform (Illumina HumanHT-12 V3.0 expression beadchip, San Diego, CA, USA) and GSE84598 is based on platform GPL10558 (Illumina HumanHT-12 V4.0 expression beadchip, San Diego, CA, USA). The platform information for GSE89377 is as follows: GPL6947, Illumina HumanHT-12 V3.0 expression beadchip (San Diego, CA, USA). A total of 196 samples were selected for differentially expressed analysis after a careful review of the sample data. As all of the data were downloaded from GEO, we did not perform any human or animal experiments.

\subsection{Differential Expression Analysis of the Selected Gene Expression Profiles}

GEO2R (https://www.ncbi.nlm.nih.gov/geo/geo2r/) [11], an interactive online analysis tool, was used to detect the differentially expressed genes (DEGs) between HCC and normal liver samples. The differential expression was analyzed separately on each profile. All parameters were set to default. The genes that met the cutoff criteria (adjusted $p$-value $<0.05$ and $\mid \log$ fold-change $(\log F C) \mid>1)$ were designated as DEGs. An online analysis tool (https://bioinfogp.cnb.csic.es/tools/venny/?/index.html) was applied to draw the Venn diagrams of up- or down-regulated genes and to detect the intersecting part of the DEGs.

\subsection{GO (Gene Ontology) and KEGG (Kyoto Encyclopedia Genes and Genomes) Enrichment Analysis of Common DEGs}

GO and KEGG pathway enrichment analyses were conducted with the intersecting DEGs. GO analysis is a regular bioinformatics method used for identifying representative biological attributes of large-scale transcriptomic or genomic data. It provides valuable information about molecular function $(\mathrm{MF})$, biological process (BP), and cellular component (CC). KEGG is a database that links DEGs with manually drawn reference pathways. The GO and KEGG pathway enrichment analyses of DEGs 
were conducted using the Database for Annotation, Visualization and Integrated Discovery, (DAVID, https://david.ncifcrf.gov/) [22]. $p<0.05$ and gene count $>10$ were the cutoff criteria for the GO and KEGG functional enrichment analysis.

\subsection{PPI Network Construction and Identification of Hub Genes}

The functional interactions between common DEGs encoded proteins may provide valuable information on molecular activities important for carcinogenesis. In this study, a protein-protein interaction (PPI) network of the common DEGs encoded proteins was constructed via the STRING database (http://string-db.org) [23]. The cut-off criterion for PPI construction was set to interaction score of $\geq 0.4$. The PPI network interaction data were then visualized with Cytoscape software (version 3.4.0, http://cytoscape.org) [11]. As a Java plugin app for Cytoscape, CytoHubba provides a user-friendly interface facilitating the analysis of complex networks with topology methods. A total of 11 topological algorithms were available for the identification of hub genes in a complex PPI network. The Maximal Clique Centrality (MCC) algorithm performs better performance in predicting hub genes in PPI networks compared with the rest of the topological algorithms. Thus, we selected the MCC algorithm to identify HCC hub genes.

\subsection{Validation of HCC Hub Gene Expression via Oncomine Database}

Oncomine is a web-based data-mining platform as well as a cancer-profiling database that aims to facilitate discovery from genome-wide expression analyses (https://www.oncomine.org) [24]. The database includes 715 gene expression data sets and gene expression data from 86,733 normal and cancer tissues. It has widely been employed in differential expression analysis or co-expression analysis between cancer and normal tissues, spanning most of the cancer types and subtypes [24,25]. Here, we obtained the mRNA expression value of the HCC hub genes based on analysis of independent gene expression profiles data with Oncomine. The gene expression data were then visualized and statistically analyzed with GraphPad software (version 5, GraphPad Software, Inc., San Diego, CA, USA).

\subsection{Survival Analysis}

The Kaplan-Meier plotter (KMplot, http://www.kmplot.com/analysis) is a database that can be used to test the role of 54,675 genes on the survival time of patients with 10,293 cancer specimens [26]. The specimens included in this database originated from 1648 ovarian, 5143 breast, 1065 gastric, 2437 lung, and 364 HCC cancer patients. The Kaplan-Meier plotter database is generally recognized as an effective approach for gene prognosis assessment in many types of cancer. In addition, the HCC genes associated survival analysis in this database is based on a paper published by Menyhárt et al. (2018) [27]. Therefore, the Kaplan-Meier plotter database is a reliable approach for the survival analysis of HCC hub genes. Here, the liver tissue mRNA RNA-seq data from the Kaplan-Meier plotter were used to assess the prognostic role of hub genes in HCC patients. For each hub gene, the specimens were divided into two groups based on the median values of mRNA expression and $p<0.05$ was considered a statistically significant result between these two groups.

\section{Results}

\subsection{Identification of DEGs with GEO2R}

The DEGs were identified by comparing HCC samples with normal liver samples. A total of 2225 DEGs were identified from GSE84598 based on the cutoff criteria of $p<0.05$ and $|\operatorname{logFC}|>1$, including 1017 up-regulated genes and 1208 down-regulated genes. For GSE87630, 1164 DEGs were screened out, including 395 up-regulated genes and 769 down-regulated genes. The GSE89377 differential expression analysis resulted in 578 DEGs with 148 up-regulated and 430 down-regulated genes. To identify the intersection among the three DEGs profiles, a Venn diagram was plotted followed 
the differential expression analysis, which resulted in 51 common up-regulated genes (Figure 1a) and 201 common down-regulated genes (Figure 1b) among GSE87630, GSE84598, and GSE89377.

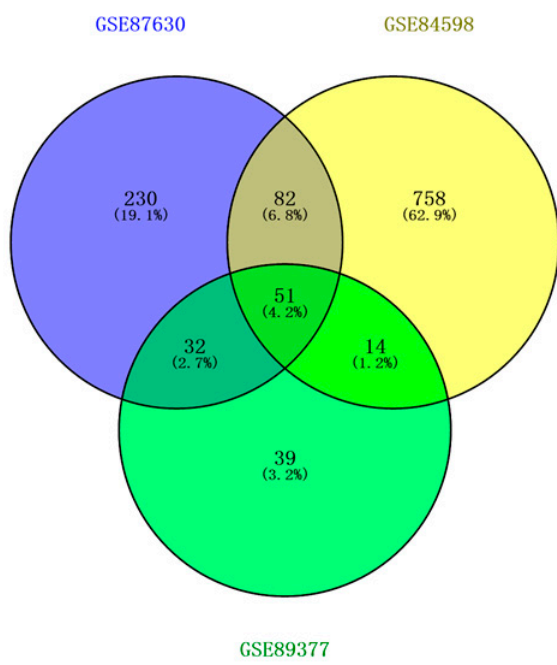

(a)

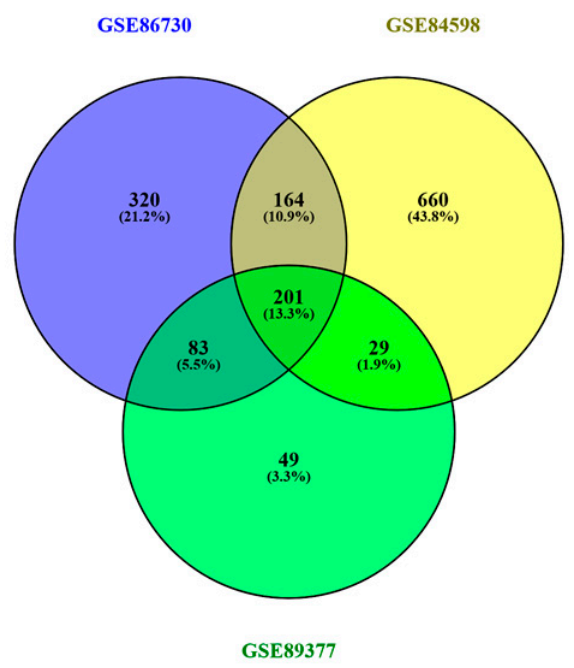

(b)

Figure 1. Venn diagram of common differentially expressed genes (DEGs) from integral analysis of three independent gene expression profiles: (a) up-regulated genes and (b) down-regulated genes from differential expression analysis of three gene expression profiles.

\subsection{GO and KEGG Enrichment Analysis}

DAVID online enrichment analysis was conducted. $n>10$ and $p<0.5$ were set as the cutoff criteria for significant enrichment. For GO BP, the common DEGs were significantly enriched in oxidation-reduction process, inflammatory response, proteolysis, immune response, cell division, complement activation, mitotic nuclear division, xenobiotic metabolic process, cell surface receptor signaling pathway, and response to drug (Figure 2a). For GO CC, the common DEGs were significantly enriched in extracellular region, organelle membrane, extracellular space, extracellular exosome, blood microparticle, perinuclear region of cytoplasm mitochondrial matrix, integral component of plasma membrane, endoplasmic reticulum membrane, and cytosol (Figure 2b). For GO MF, the common DEGs were significantly enriched in oxidoreductase activity, monooxygenase activity, heme binding, iron ion binding, serine-type endopeptidase activity, enzyme binding, receptor binding, calcium ion binding, and protein homodimerization activity (Figure 2c). As shown in Figure 2d, the most significantly enriched KEGG pathways included cell cycle, metabolic pathways, chemical carcinogenesis, and complement, and coagulation cascades. 


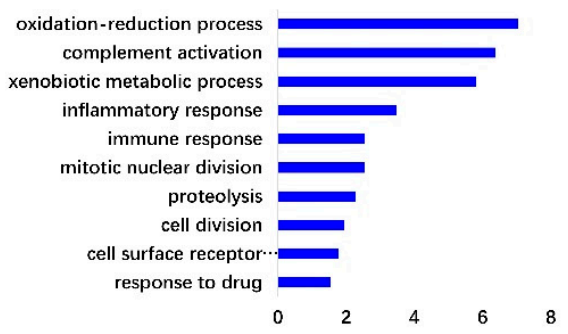

(a)

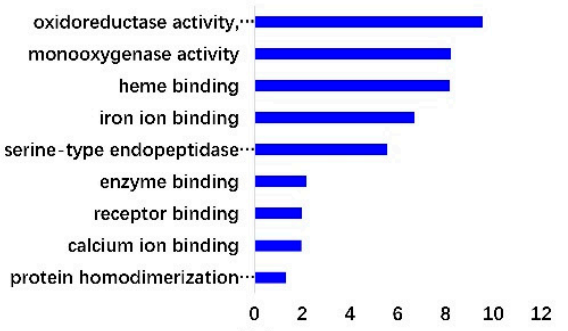

(c)

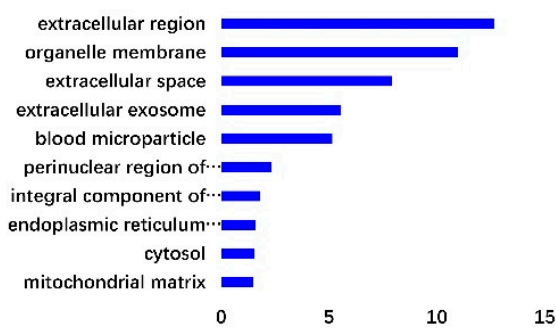

(b)

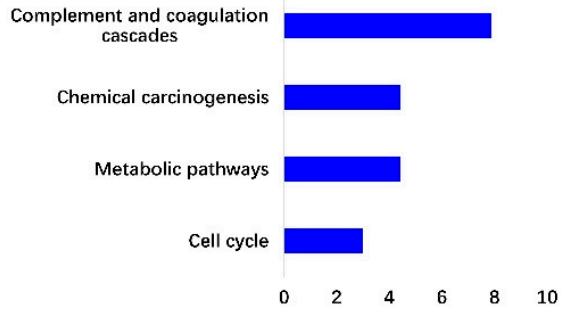

(d)

Figure 2. The significantly enriched Gene Ontology (GO) terms and Kyoto Encyclopedia Genes and Genomes (KEGG) pathways of hepatocellular carcinoma (HCC) differentially expressed genes. (a) GO biological process, (b) GO cellular component, and (c) GO molecular function enrichment analysis results of DEGs. (d) GO KEGG pathway enrichment analysis of DEGs in HCC.

\subsection{PPI Network Construction and Identification of Hub Genes Based on Network Topological Analysis}

The 252 common DEGs of the results from the differential expression analysis of the HCC gene expression profiles were used to construct a PPI network with the STRING database. The constructed PPI network includes a total of 250 nodes and 887 edges. CytoHubba, an app for Cytoscape software, is generally used to predict hub nodes from a given network based on 11 topological algorithms. Here, the Maximal Clique Centrality (MCC) algorithm of CytoHubba was employed to identify the hub genes. A total of 10 hub genes were finally identified: CDCA5, OIP5, TOP2A, PRC1, ASPM, NUSAP1, UBE2C, CDC20, CCNA2, and KIF20A (Figure 3). LogFC is the log difference of actual expression values results from expression analysis of two groups of tissue samples. The logFC of the HCC hub genes is displayed in Table 1, revealing the expression alterations of hub genes in HCC samples compared with normal liver tissue. A positive logFC indicates up-regulation of the hub genes, whereas a negative $\log$ FC suggests down-regulation of genes. All the logFC values of the HCC hub genes were positive, indicating an overexpression of the hub genes in HCC tissues. 


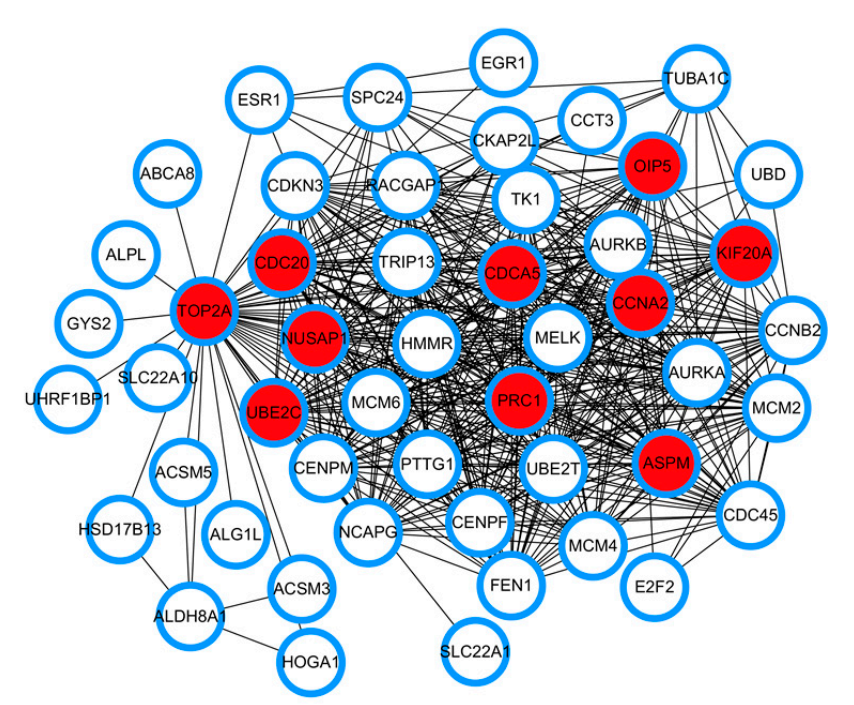

Figure 3. The protein-protein interaction network of the hub genes and the DEGs that directly interact with them. Each node represents a gene and each edge represents a direct interaction between two genes. The nodes filled with red in the center represent hub genes; the nodes filled with white represent the genes that interact directly with hub genes.

Table 1. Log fold-change ( $\log \mathrm{FC}$ ) of hub gene expression values in HCC samples compared with normal samples.

\begin{tabular}{cccc}
\hline Hub Genes & GSE87630 & GSE89377 & GSE84598 \\
\hline CDCA5 & 2.28 & 1.9 & 2.55 \\
OIP5 & 1.32 & 1.29 & 1.72 \\
TOP2A & 3.15 & 2.33 & 3.21 \\
PRC1 & 2.74 & 1.81 & 2.56 \\
ASPM & 1.53 & 1.61 & 2.25 \\
NUSAP1 & 1.82 & 1.67 & 1.53 \\
UBE2C & 1.88 & 2.4 & 2.77 \\
CDC20 & 3.01 & 2.33 & 3.09 \\
CCNA2 & 1.56 & 1.03 & 1.82 \\
KIF20A & 2.22 & 1.37 & 3.76 \\
\hline
\end{tabular}

\subsection{Validation of Hub Genes Expression Level with Data Mining Using Oncomine Database}

Based on data mining of the GSE6764 dataset from the Oncomine database, the gene expression values of ASPM, CCNA2, CDC20, CDCA5, KIF20A, and OIP5 were obtained. Similarly, the gene expression values of NUSAP1, PRC1, TOP2A, and UBE2C were also obtained via data mining of the GSE14520 dataset from the Oncomine database. The gene expression values of the hub genes were then statistically analyzed and visualized with GraphPad software 5.0 (San Diego, CA, USA). Our results indicated that the HCC tissues have significantly higher levels of mRNA expression in terms of hub genes compared to normal liver tissues (Student's $t$-test, $p<0.05$; Figure $4 \mathrm{a}-\mathrm{j}$ ). 


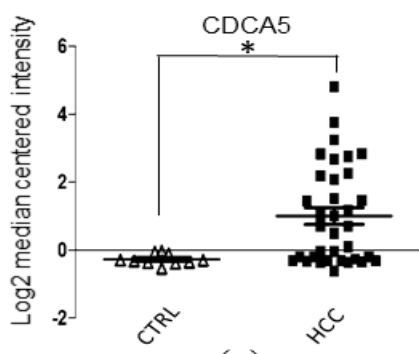

(a)

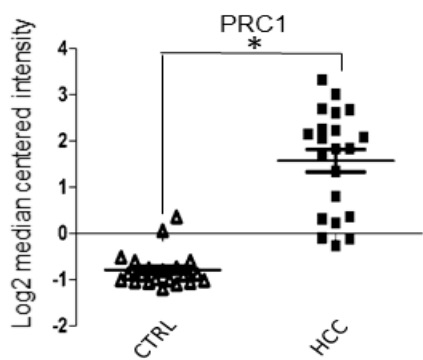

(d)

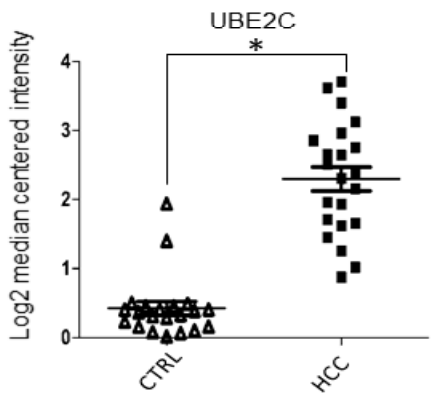

(g)

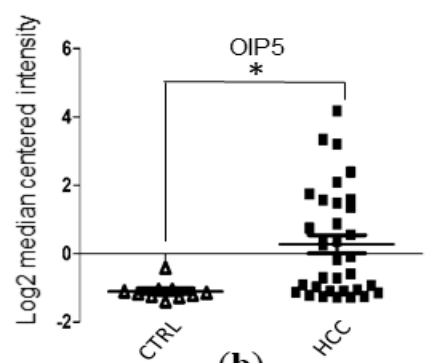

(b)

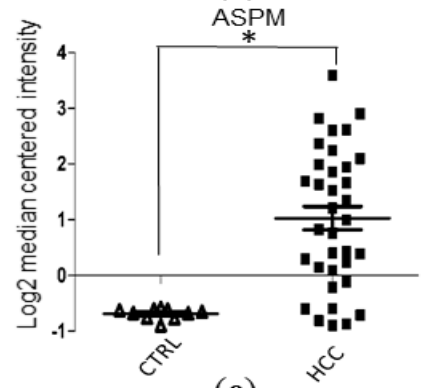

(e)

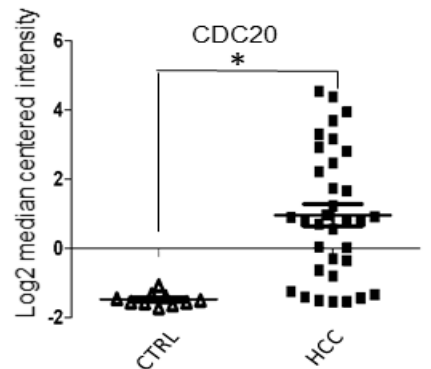

(h)

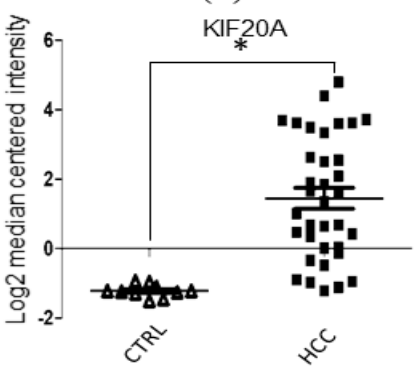

(j)

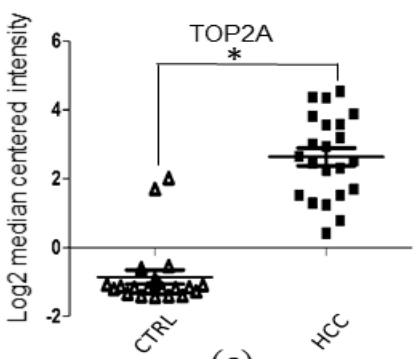

(c)

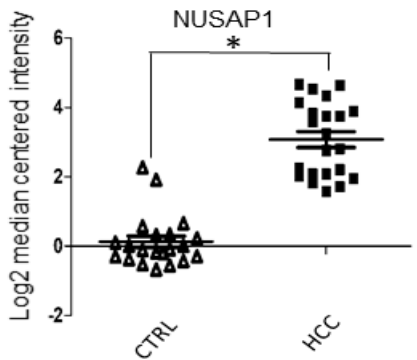

(f)

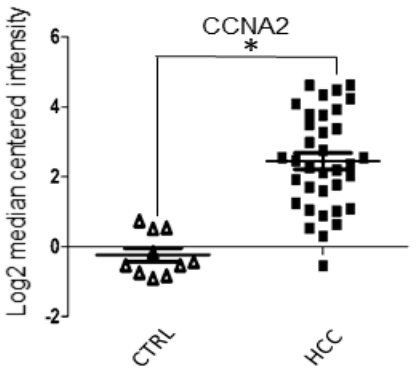

(i)

Figure 4. Validation of HCC hub gene expression levels with data mining of the Oncomine database: $(\mathbf{a}-\mathbf{j})$ The gene expression level of the hub genes between HCC tissues and normal liver tissues. *, a significant statistical difference was observed between HCC and normal liver tissues. Based on the analysis of the GSE6764 dataset, ASPM, CCNA2, CDC20, CDCA5, KIF20A, and OIP5 have significantly higher levels of expression in HCC samples compared to normal liver tissues (Student's $t$-test, $p<0.05$ ). Similarly, NUSAP1, PRC1, TOP2A, and UBE2C have significantly higher levels of expression in HCC samples compared to normal liver tissues (Student's $t$-test, $p<0.05$ ) via analysis of GSE14520 dataset with the Oncomine database (Student's $t$-test, $p<0.05$ ).

\subsection{Survival Analysis}

The Kaplan-Meier plotter was used to evaluate the role of the identified hub genes on the prognosis of HCC. A total of 364 liver samples were available for current survival analysis. We found that overexpression of these hub genes is associated with a significant reduction in survival time of HCC patients $(p<0.05$; Figure $5 a-j)$. 


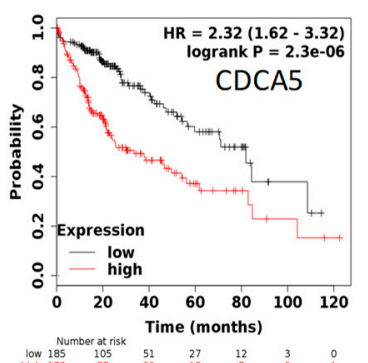

(a)

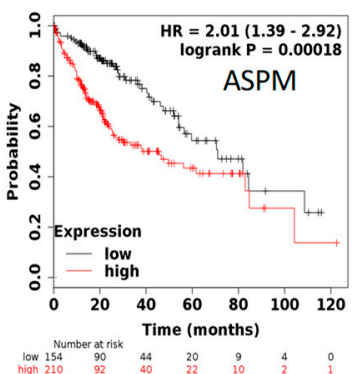

(e)

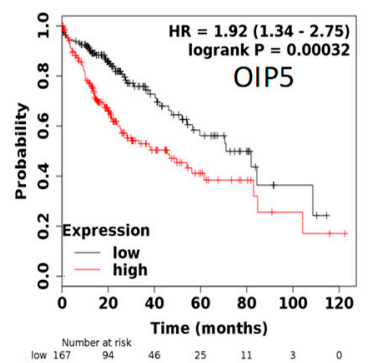

(b)

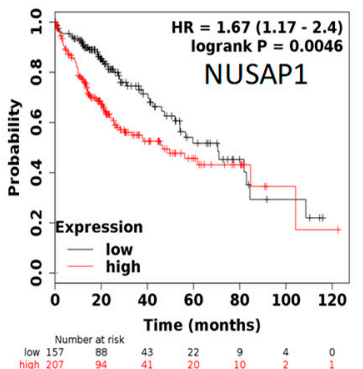

(f)

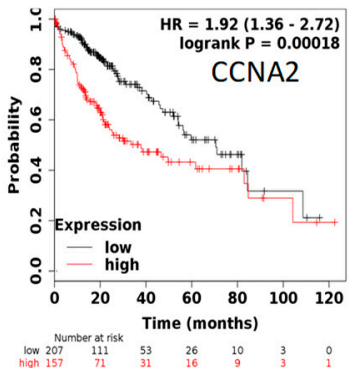

(i)

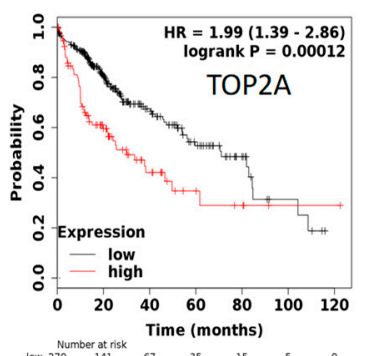

(c)

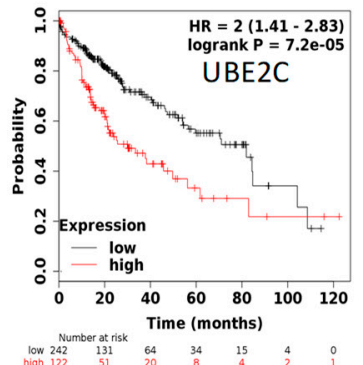

(g)

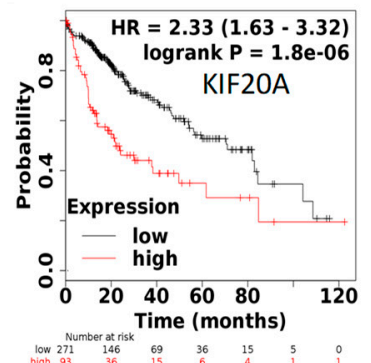

(j)

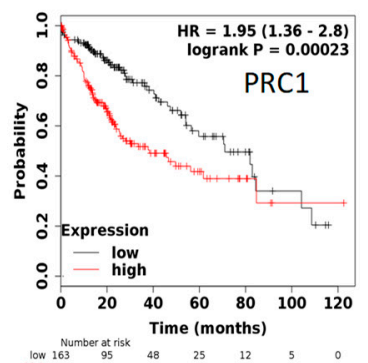

(d)

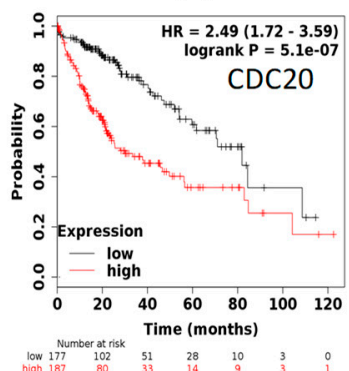

(h)

Figure 5. Kaplan-Meier survival analyses of the HCC hub genes. (a-j) Overexpression of these HCC hub genes is associated with a significant reduction in overall survival time in HCC patients.

\section{Discussion}

HCC, a major health threat to people worldwide, is one of the leading causes of cancer mortality [28]. It occurs more frequently among men than women, with increasing incidence rates in almost all countries, particularly in East Asia [29]. The etiologies of HCC include metabolic diseases [30], alcohol-related cirrhosis [31], hepatitis B virus (HBV) [32], and hepatitis C virus (HCV) [33]. HCV-associated hepatitis develops into HCC via a myc-unregulated mechanism [34], whereas non-alcoholic steatohepatitis-dependent disease is not involved in the expression of myc [35]. The large difference in etiologies might contribute to the heterogeneity of HCC.

So far, no targeted therapies have been proven to be effective against HCC. Due to the heterogeneity of HCC, the current targeted cancer drugs only selectively kill some tumor cells, leaving a small part of the surviving tumor cells almost unaffected, which gradually develop into the main body of tumor [36]. Therefore, HCC studies should cover as many cases and genes as possible and this increase in sample size or gene numbers may allow us to detect a large number of genes highly associated with HCC.

The development of microarray technology has allowed to measure numerous gene expression aberrations simultaneously. Currently, many gene expression profiles are freely available in GEO. In this study, three gene expression profiles were selected from GEO for differential expression analysis. Venn analysis resulted in a total of 51 common up-regulated genes and 201 down-regulated genes. GO enrichment analyses indicated that the common DEGs are closely linked to a series of cancer functional events. The function enriched KEGG pathways are associated with cell cycle. The top 10 hub genes include CDCA5, OIP5, TOP2A, PRC1, ASPM, NUSAP1, UBE2C, CDC20, CCNA2, 
and KIF20A. Cai et al. showed that CDC20, AURKB, BIRC5, RRM2, MCM2, PTTG1, CDKN2A, NEK2, $C E N P F, R A C G A P 1, G N A 14$, and $C D C A 5$ are hub genes that may be important for diagnosis, clinical intervention, and prognosis of HCC [37]. TOP2A, NDC80, FOXM1, HMMR, KNTC1, PTTG1, FEN1, RFC4, SMC4, and PRC1 were reported as being the top 10 core HCC genes in another study, sharing two genes with our study [38]. Heterogeneity, sample size, and methods of identifying hub genes may contribute to the differences in hub genes. Six of the hub genes (OIP5, ASPM, NUSAP1, UBE2C, CCNA2, and KIF20A) were identified for the first time in comparison with previous reports.

In the current study, we combined three gene expression datasets to find the hub genes important for HCC progression or prognosis. Three gene expression profiles from different regions were used and the sample size was larger compared to some of the previous studies, which might have helped to minimize the adverse effects caused by heterogeneity and small sample size. Cell-division cycle-associated 5 (CDCA5) is believed to play an essential role in the accurate separation of sister chromatids throughout the $\mathrm{S}$ and G2/M phases of the cell cycle $[39,40]$. A clinical study confirmed CDCA5 overexpression is associated with poor prognosis in patients with HCC [41]. Opa interacting protein 5 (OIP5) is reported to regulate growth and metastasis of HCC via the Protein Kinase B $(\mathrm{AKT}) /$ mammalian target of the rapamycin (mTOR) signaling pathways [42]. High expression of DNA topoisomerase II alpha (TOP2A) was detected in $72.5 \%$ of HCC tumor tissues [43] and an increase in TOP2A expression is correlated with shorter survival of patients and chemoresistance [44]. Protein regulator of cytokinesis 1(PRC1) overexpression in HCC cells can cause enhanced chemoresistance and attenuation of apoptosis for patients who received chemotherapy [45]. Abnormal spindle-like microcephaly-associated protein (ASPM) overexpression has been shown to be a molecular marker predicting enhanced metastatic potential of HCC with poor prognosis [46]. Of the HCC patients, $54.1 \%$ were observed to have high expression of nucleolar- and spindle-associated protein 1 (NUSAP1) and this overexpression of NUSAP1 is associated with a significantly lower survival rate of patients [47]. microRNA 193a-5p was proven to suppress mice hepatocarcinogenesis via the regulation of NUSAP1 level [48]. So far, little information is available regarding the contribution of ubiquitin conjugating enzyme E2 C (UBE2C) to HCC development. Up-regulation of cell division cycle 20 (CDC20) in tumor tissues can be used to predict worse overall survival in HCC patients [49]. In vitro experiments showed cyclin A2 (CCNA2) contributes to the tumorigenesis of HCC [50]. In comparison with normal liver tissues, HCC tissues generally have high levels of kinesin family member 20A (KIF20A) expression with worse survival outcomes [51]. Overall, most of the hub genes identified in this study had a high level of expression with poor prognosis.

To further validate the expression level of the hub genes, we performed Oncomine-database-based mRNA expression-level analysis of the hub genes which showed that all the hub genes have significantly higher levels of expression in comparison with normal liver tissues. The results suggest that the hub genes we screened out are reliable biomarkers of HCC.

Survival analysis showed that high expression of these hub genes is associated with a significant reduction $(p<0.05)$ in overall survival time in HCC patients. Overexpression of these hub genes, especially the six hub genes with no previous reports, may serve as unfavorable prognostic factors for HCC patients.

\section{Conclusions}

In this study, $10 \mathrm{HCC}$ hub genes were identified, with few reports of six of them. Overexpression of the hub genes is significantly associated with decreased survival time in HCC patients. The hub genes play a role in disease onset or progression, which might be novel therapeutic targets for the treatment of HCC for patients with similar genetic alterations.

Author Contributions: Conceptualization: C.L. and J.X.; methodology: C.L.; validation: C.L. and J.X.; formal analysis: C.L.; data curation: C.L.; writing—original draft preparation: C.L.; writing—review and editing: J.X.; visualization: C.L.; supervision: J.X.; project administration: J.X.; funding acquisition: J.X. All authors have read and agreed to the published version of the manuscript. 
Funding: This research was funded by National Natural Science Foundation of China, grant numbers 61976082,61772176, 61370169, 61402153; the Plan for Scientific Innovation Talent of Henan Province, grant number 184100510003; the Project of Science and Technology Department of Henan Province of China, grant number 182102210362; the Young Scholar Program of Henan Province, grant number 2017GGJS041; and the Key Scientific and Technological Project of Xinxiang City of China grant number CXGG17002.

Acknowledgments: We thank Wang Nan, Wang Yun, Xu Keqiang, Yang Lulu, and Cheng Yihao their helpful advice to improve the quality of this paper.

Conflicts of Interest: The authors declare no conflict of interest. The funders had no role in the design of the study; in the collection, analyses, or interpretation of data; in the writing of the manuscript; or in the decision to publish the results.

\section{References}

1. $\quad$ Li, S.-Q.; Chen, Q.; Qin, H.-X.; Yu, Y.-Q.; Weng, J.; Mo, Q.-R.; Yin, X.-F.; Lin, Y.; Liao, W.-J. LINC00152 promotes hepatocellular carcinoma progression by regulating PI3k/Akt/mTOR signaling pathway through miR-139/PIK3CA. Am. J. Pathol. 2020. [CrossRef] [PubMed]

2. Sun, V.C.; Sarna, L. Symptom management in hepatocellular carcinoma. Clin. J. Oncol. Nurs. 2008, 12, 759-766. [CrossRef] [PubMed]

3. Bertuccio, P.; Turati, F.; Carioli, G.; Rodriguez, T.; La Vecchia, C.; Malvezzi, M.; Negri, E. Global trends and predictions in hepatocellular carcinoma mortality. J. Hepatol. 2017, 67, 302-309. [CrossRef] [PubMed]

4. Mazzaferro, V.; Gorgen, A.; Roayaie, S.; Droz Dit Busset, M.; Sapisochin, G. Liver resection and transplantation for intrahepatic cholangiocarcinoma. J. Hepatol. 2020, 72, 364-377. [CrossRef] [PubMed]

5. Huo, Y.R.; Chan, M.V.; Chan, C. Resection plus post-operative adjuvant transcatheter arterial chemoembolization (tace) compared with resection alone for hepatocellular carcinoma: A systematic review and meta-analysis. Cardiovasc. Intervent. Radiol. 2020. [CrossRef] [PubMed]

6. Gui, C.H.; Baey, S.; D'Cruz, R.T.; Shelat, V.G. Trans-arterial chemoembolization radiofrequency ablation versus surgical resection in hepatocellular carcinoma-A meta-analysis. Eur. J. Surg. Oncol. 2020. [CrossRef] [PubMed]

7. Bhutiani, N.; O’Brien, S.J.; Priddy, E.E.; Egger, M.E.; Hong, Y.K.; Mercer, M.K.; McMasters, K.M.; Martin, R.C.G.; Potts, M.H.; Scoggins, C.R. Correlating serum alpha-fetoprotein in hepatocellular carcinoma with response to Yttrium-90 transarterial radioembolization with glass microspheres (TheraSphere ${ }^{\mathrm{TM}}$ ). HPB 2020. [CrossRef]

8. Xu, Y.; Kong, Y.; Xu, J.; Li, X.; Gou, J.; Yin, T.; He, H.; Zhang, Y.; Tang, X. Doxorubicin intercalated copper diethyldithiocarbamate functionalized layered double hydroxide hybrid nanoparticles for targeted therapy of hepatocellular carcinoma. Biomater. Sci. 2019. [CrossRef]

9. Lee, G.C.; Ferrone, C.R.; Vagefi, P.A.; Uppot, R.N.; Tanabe, K.K.; Lillemoe, K.D.; Blaszkowsky, L.S.; Qadan, M. Surgical resection versus ablation for early-stage hepatocellular carcinoma: A retrospective cohort analysis. Am. J. Surg. 2019. [CrossRef]

10. Pan, Y.-X.; Chen, J.-C.; Fang, A.-P.; Wang, X.-H.; Chen, J.-B.; Wang, J.-C.; He, W.; Fu, Y.-Z.; Xu, L.; Chen, M.-S.; et al. A nomogram predicting the recurrence of hepatocellular carcinoma in patients after laparoscopic hepatectomy. Cancer Commun. 2019, 39, 55. [CrossRef]

11. Zhang, C.; Peng, L.; Zhang, Y.; Liu, Z.; Li, W.; Chen, S.; Li, G. The identification of key genes and pathways in hepatocellular carcinoma by bioinformatics analysis of high-throughput data. Med. Oncol. 2017, 34, 101. [CrossRef] [PubMed]

12. Mittal, S.; Kramer, J.R.; Omino, R.; Chayanupatkul, M.; Richardson, P.A.; El-Serag, H.B.; Kanwal, F. Role of age and race in the risk of hepatocellular carcinoma in veterans with hepatitis b virus infection. Clin. Gastroenterol. Hepatol. 2018, 16, 252-259. [CrossRef] [PubMed]

13. Kamp, W.M.; Sellers, C.M.; Stein, S.; Lim, J.K.; Kim, H.S. Impact of direct acting antivirals on survival in patients with chronic hepatitis c and hepatocellular carcinoma. Sci. Rep. 2019, 9, 17081. [CrossRef] [PubMed]

14. Yang, T.; Xing, H.; Wang, G.; Wang, N.; Liu, M.; Yan, C.; Li, H.; Wei, L.; Li, S.; Fan, Z.; et al. A novel online calculator based on serum biomarkers to detect hepatocellular carcinoma among patients with Hepatitis B. Clin. Chem. 2019, 65, 1543-1553. [CrossRef]

15. Wang, L.; Li, H.; Zhen, Z.; Ma, X.; Yu, W.; Zeng, H.; Li, L. CXCL17 promotes cell metastasis and inhibits autophagy via the LKB1-AMPK pathway in hepatocellular carcinoma. Gene 2019, 690, 129-136. [CrossRef] [PubMed] 
16. Tan, W.; Luo, X.; Li, W.; Zhong, J.; Cao, J.; Zhu, S.; Chen, X.; Zhou, R.; Shang, C.; Chen, Y. TNF-alpha is a potential therapeutic target to overcome sorafenib resistance in hepatocellular carcinoma. EBioMedicine 2018. [CrossRef]

17. Chen, P.F.; Li, Q.H.; Zeng, L.R.; Yang, X.Y.; Peng, P.L.; He, J.H.; Fan, B. A 4-gene prognostic signature predicting survival in hepatocellular carcinoma. J. Cell. Biochem. 2018. [CrossRef]

18. Cao, J.; Bu, Z.; Wang, Y.; Yang, H.; Jiang, J.; Li, H. Detecting prosumer-community groups in smart grids from the multiagent perspective. IEEE Trans. Syst. Man Cybern. Syst. 2019, 49, 1652-1664. [CrossRef]

19. Bu, Z.; Wang, Y.; Li, H.; Jiang, J.; Wu, Z.; Cao, J. Link prediction in temporal networks: Integrating survival analysis and game theory. Inf. Sci. 2019, 498, 41-61. [CrossRef]

20. Song, A.; Liu, Y.; Wu, Z.; Zhai, M.; Luo, J. A local random walk model for complex networks based on discriminative feature combinations. Expert Syst. Appl. 2019, 118, 329-339. [CrossRef]

21. Hu, W.Q.; Wang, W.; Fang, D.L.; Yin, X.F. Identification of biological targets of therapeutic intervention for hepatocellular carcinoma by integrated bioinformatical analysis. Med. Sci. Monit. 2018, 24, 3450-3461. [CrossRef] [PubMed]

22. Huang, D.W.; Sherman, B.T.; Lempicki, R.A. Bioinformatics enrichment tools: paths toward the comprehensive functional analysis of large gene lists. Nucleic Acids Res 2009, 37, 1-13. [CrossRef] [PubMed]

23. Szklarczyk, D.; Gable, A.L.; Lyon, D.; Junge, A.; Wyder, S.; Huerta-Cepas, J.; Simonovic, M.; Doncheva, N.T.; Morris, J.H.; Bork, P.; et al. STRING v11: Protein-protein association networks with increased coverage, supporting functional discovery in genome-wide experimental datasets. Nucleic. Acids Res. 2019, 47, D607-D613. [CrossRef] [PubMed]

24. Rhodes, D.R.; Kalyana-Sundaram, S.; Mahavisno, V.; Varambally, R.; Yu, J.; Briggs, B.B.; Barrette, T.R.; Anstet, M.J.; Kincead-Beal, C.; Kulkarni, P.; et al. Oncomine 3.0: Genes, pathways, and networks in a collection of 18,000 cancer gene expression profiles. Neoplasia 2007, 9, 166-180. [CrossRef]

25. Rhodes, D.R.; Yu, J.; Shanker, K.; Deshpande, N.; Varambally, R.; Ghosh, D.; Barrette, T.; Pandey, A.; Chinnaiyan, A.M. ONCOMINE: A cancer microarray database and integrated data-mining platform. Neoplasia 2004, 6, 1-6. [CrossRef]

26. Nagy, Á.; Lánczky, A.; Menyhárt, O.; Győrffy, B. Author Correction: Validation of miRNA prognostic power in hepatocellular carcinoma using expression data of independent datasets. Sci. Rep. 2018, 8, 11515. [CrossRef]

27. Menyhárt, O.; Nagy, Á.; Győrffy, B. Determining consistent prognostic biomarkers of overall survival and vascular invasion in hepatocellular carcinoma. R. Soc. Open Sci. 2018, 5, 181006. [CrossRef]

28. Bruix, J.; Gores, G.J.; Mazzaferro, V. Hepatocellular carcinoma: Clinical frontiers and perspectives. Gut 2014, 63, 844-855. [CrossRef]

29. Lin, D.C.; Mayakonda, A.; Dinh, H.Q.; Huang, P.; Lin, L.; Liu, X.; Ding, L.W.; Wang, J.; Berman, B.P.; Song, E.W.; et al. Genomic and epigenomic heterogeneity of hepatocellular carcinoma. Cancer Res. 2017, 77, 2255-2265. [CrossRef]

30. Baffy, G. Hepatocellular carcinoma in obesity: Finding a needle in the haystack? Adv. Exp. Med. Biol. 2018, 1061, 63-77. [CrossRef]

31. Stickel, F.; Buch, S.; Nischalke, H.D.; Weiss, K.H.; Gotthardt, D.; Fischer, J.; Rosendahl, J.; Marot, A.; Elamly, M.; Casper, M.; et al. Genetic variants in PNPLA3 and TM6SF2 predispose to the development of hepatocellular carcinoma in individuals with alcohol-related cirrhosis. Am. J. Gastroenterol. 2018, 113, 1475-1483. [CrossRef] [PubMed]

32. Zhu, G.-Z.; Liao, X.-W.; Wang, X.-K.; Gong, Y.-Z.; Liu, X.-G.; Yu, L.; Han, C.-Y.; Yang, C.-K.; Su, H.; Huang, K.-T.; et al. Comprehensive investigation of p53, p21, nm23, and VEGF expression in hepatitis B virus-related hepatocellular carcinoma overall survival after hepatectomy. J. Cancer 2020, 11, 906-918. [CrossRef] [PubMed]

33. El-Maraghy, S.A.; Adel, O.; Zayed, N.; Yosry, A.; El-Nahaas, S.M.; Gibriel, A.A. Circulatory miRNA-484, 524,615 and 628 expression profiling in HCV mediated HCC among Egyptian patients; implications for diagnosis and staging of hepatic cirrhosis and fibrosis. J. Adv. Res. 2020, 22, 57-66. [CrossRef] [PubMed]

34. Koutb, F.; Abdel-Rahman, S.; Hassona, E.; Haggag, A. Association of c-myc and p53 gene expression and polymorphisms with hepatitis c (hcv) chronic infection, cirrhosis and hepatocellular carcinoma (hcc) stages in egypt. Asian Pac. J. Cancer Prev. 2017, 18, 2049-2057. [CrossRef] 
35. Fransvea, E.; Paradiso, A.; Antonaci, S.; Giannelli, G. HCC heterogeneity: Molecular pathogenesis and clinical implications. Cell. Oncol. 2009, 31, 227-233. [CrossRef]

36. Jeng, K.S.; Chang, C.F.; Jeng, W.J.; Sheen, I.S.; Jeng, C.J. Heterogeneity of hepatocellular carcinoma contributes to cancer progression. Crit. Rev. Oncol./Hematol. 2015, 94, 337-347. [CrossRef]

37. Cai, C.; Wang, W.; Tu, Z. Aberrantly DNA methylated-differentially expressed genes and pathways in hepatocellular carcinoma. J. Cancer 2019, 10, 355-366. [CrossRef]

38. Shen, S.; Kong, J.; Qiu, Y.; Yang, X.; Wang, W.; Yan, L. Identification of core genes and outcomes in hepatocellular carcinoma by bioinformatics analysis. J. Cell. Biochem. 2018. [CrossRef]

39. Borton, M.T.; Rashid, M.S.; Dreier, M.R.; Taylor, W.R. Multiple levels of regulation of sororin by cdk1 and aurora B. J. Cell. Biochem. 2016, 117, 351-360. [CrossRef]

40. Schmitz, J.; Watrin, E.; Lenart, P.; Mechtler, K.; Peters, J.M. Sororin is required for stable binding of cohesin to chromatin and for sister chromatid cohesion in interphase. Curr. Biol. 2007, 17, 630-636. [CrossRef]

41. Tian, Y.; Wu, J.; Chagas, C.; Du, Y.; Lyu, H.; He, Y.; Qi, S.; Peng, Y.; Hu, J. CDCA5 overexpression is an Indicator of poor prognosis in patients with hepatocellular carcinoma (HCC). BMC Cancer 2018, 18, 1187. [CrossRef]

42. Li, H.; Zhang, J.; Lee, M.J.; Yu, G.R.; Han, X.; Kim, D.G. OIP5, a target of miR-15b-5p, regulates hepatocellular carcinoma growth and metastasis through the AKT/mTORC1 and beta-catenin signaling pathways. Oncotarget 2017, 8, 18129-18144. [CrossRef] [PubMed]

43. Panvichian, R.; Tantiwetrueangdet, A.; Angkathunyakul, N.; Leelaudomlipi, S. TOP2A amplification and overexpression in hepatocellular carcinoma tissues. BioMed. Res. Int. 2015, 2015, 381602. [CrossRef] [PubMed]

44. Wong, N.; Yeo, W.; Wong, W.L.; Wong, N.L.; Chan, K.Y.; Mo, F.K.; Koh, J.; Chan, S.L.; Chan, A.T.; Lai, P.B.; et al. TOP2A overexpression in hepatocellular carcinoma correlates with early age onset, shorter patients survival and chemoresistance. Int. J. Cancer 2009, 124, 644-652. [CrossRef]

45. Wang, Y.; Shi, F.; Xing, G.H.; Xie, P.; Zhao, N.; Yin, Y.F.; Sun, S.Y.; He, J.; Wang, Y.; Xuan, S.Y. Protein regulator of cytokinesis prc1 confers chemoresistance and predicts an unfavorable postoperative survival of hepatocellular carcinoma patients. J. Cancer 2017, 8, 801-808. [CrossRef] [PubMed]

46. Lin, S.Y.; Pan, H.W.; Liu, S.H.; Jeng, Y.M.; Hu, F.C.; Peng, S.Y.; Lai, P.L.; Hsu, H.C. ASPM is a novel marker for vascular invasion, early recurrence, and poor prognosis of hepatocellular carcinoma. Clin. Cancer Res. 2008, 14, 4814-4820. [CrossRef] [PubMed]

47. Zhang, M.; Yang, D.; Liu, X.; Liu, Y.; Liang, J. Expression and clinical significance of Nusap1 in hepatical carcinoma. J. Cent. South Univ. Med. Sci. 2013, 38, 876-881. [CrossRef]

48. Roy, S.; Hooiveld, G.J.; Seehawer, M.; Caruso, S.; Heinzmann, F.; Schneider, A.T.; Frank, A.K.; Cardenas, D.V.; Sonntag, R.; Luedde, M.; et al. microRNA 193a-5p regulates levels of nucleolar- and spindle-associated protein 1 to suppress hepatocarcinogenesis. Gastroenterology 2018, 155, 1951-1966. [CrossRef]

49. Zhuang, L.; Yang, Z. Upregulation of BUB1B, CCNB1, CDC7, CDC20, and MCM3 in tumor tissues predicted worse overall survival and disease-free survival in hepatocellular carcinoma patients. Biomed. Res. Int. 2018, 2018, 7897346. [CrossRef]

50. Yang, F.; Gong, J.; Wang, G.; Chen, P.; Yang, L.; Wang, Z. Waltonitone inhibits proliferation of hepatoma cells and tumorigenesis via FXR-miR-22-CCNA2 signaling pathway. Oncotarget 2016, 7, 75165-75175. [CrossRef]

51. Lu, M.; Huang, X.; Chen, Y.; Fu, Y.; Xu, C.; Xiang, W.; Li, C.; Zhang, S.; Yu, C. Aberrant KIF20A expression might independently predict poor overall survival and recurrence-free survival of hepatocellular carcinoma. IUBMB Life 2018, 70, 328-335. [CrossRef] [PubMed]

(C) 2020 by the authors. Licensee MDPI, Basel, Switzerland. This article is an open access article distributed under the terms and conditions of the Creative Commons Attribution (CC BY) license (http://creativecommons.org/licenses/by/4.0/). 